\title{
Exploring Planets with Directed Aerial Robot Explorers
}

\author{
Alexey A. Pankine ${ }^{1}$, Kim M. Aaron ${ }^{1}$, Matthew K. Heun ${ }^{1}$, Kerry T. Nock ${ }^{1}$, \\ R. Stephen Schlaifer ${ }^{1}$, Andrew P. Ingersoll ${ }^{2}$, and Ralph D. Lorenz ${ }^{3}$ \\ ${ }^{1}$ Global Aerospace Corporation, 711 W. Woodbury Rd., Suite H, Altadena, CA 91001, USA \\ ${ }^{2}$ California Institute of Technology, 1200 E. California Blvd., Pasadena, CA 91125, USA \\ ${ }^{3}$ Lunar and Planetary Laboratory, 1629 E. University Blvd., Tucson, AZ 85721, USA \\ 626-345-1200,alexey.a.pankine@gaerospace.com
}

\begin{abstract}
Global Aerospace Corporation (GAC) is developing a revolutionary system architecture for exploration of planetary atmospheres and surfaces from atmospheric altitudes. The work is supported by the NASA Institute for Advanced Concepts (NIAC). The innovative system architecture relies upon the use of Directed Aerial Robot Explorers (DAREs), which essentially are long-duration-flight autonomous balloons with trajectory control capabilities that can deploy swarms of miniature probes over multiple target areas. Balloon guidance capabilities will offer unprecedented opportunities in high-resolution, targeted observations of both atmospheric and surface phenomena. Multifunctional microprobes will be deployed from the balloons once over the target areas, and perform a multitude of functions, such as atmospheric profiling or surface exploration, relaying data back to the balloons or an orbiter. This architecture will enable low-cost, low-energy, long-term global exploration of planetary atmospheres and surfaces. This paper focuses on a conceptual analysis of the DARE architecture capabilities and science applications for Venus, Titan and Jupiter. Preliminary simulations with simplified atmospheric models show that a relatively small trajectory control wing can enable global coverage of the atmospheres of Venus and Titan by a single balloon over a 100-day mission. This presents unique opportunities for global in situ sampling of the atmospheric composition and dynamics, atmospheric profiling over multiple sites with small dropsondes and targeted deployment of surface microprobes. At Jupiter, path guidance capabilities of the DARE platforms permits targeting localized regions of interest, such as "hot spots" or the Great Red Spot. A single DARE platform at Jupiter can sample major types of the atmospheric flows (zones and belts) over a 100-day mission. Observations by deployable probes would reveal if the differences exist in radiative, dynamic and compositional environments at these sites.
\end{abstract}

\section{INTRODUCTION}

Balloons have been long recognized as unique scientific platforms due to their relatively low cost and low power consumption. Indeed, the successful Venera-Vega Project (Sagdeev et al., 1986) demonstrated technical feasibility of deploying a balloon on another planet and performing scientific observations from it. Concepts and technologies enabling planetary balloon exploration of Venus, Titan, Mars, and the Outer Planets have been developed (Bachelder et al., 1999; Cutts et al., 1999; Greeley et al., 1996; Jones and Wu, 1999; Jones and Heun, 1997; JPL, 1997; Nock et al., 1997; SAIC, 1983; Tarrieu, 1993). The DARE architecture advances these concepts to the next level of utility and universality by integrating the balloon platform with the innovative lightweight Balloon Guidance System (BGS) and multiple lightweight deployable microprobes into a revolutionary architecture for planetary exploration. This architecture would greatly expand the planetary exploration capabilities making possible high-resolution targeted observations, and augmenting observations at atmospheric altitudes with in situ surface observations.

DARE represents a highly adaptive observational platform capable of observing planetary atmospheres and surfaces over long periods of time without consuming much power. The DARE platforms would orbit the planet using winds to guide their trajectory according to observational objectives. Studies of the atmospheric dynamics, atmospheric chemical, and radiative processes on other planets would become possible at an advanced level. Small microprobes would be deployed over the target areas and perform a multitude of tasks at the surface or while descending, such as chemical, biological, meteorological, or thermal analyses, high-resolution imaging, measuring seismic activity, etc. The data would be transmitted in real time to the overflying DARE platform, processed or temporarily stored 
onboard, and then relayed to the orbiter, or transmitted to the orbiter directly. Some deployable microprobes could be capable of descending to the surface, "grabbing" a sample of the surface material and then ascending back to the altitude of the DARE platform to rendezvous with the platform and transfer the sample for geochemical analysis onboard the platform.

The key elements of the overall DARE architecture are: 1) long-duration planetary balloon; 2) light-weight balloon trajectory control; 3) lightweight and efficient power generation and energy storage; 4) multiple deployable microprobes; and 5) communication relay orbiter. Below we overview the DARE architecture and give examples of applications at Venus, Titan and Jupiter. DARE architecture for Mars is described in the accompanying paper (Pankine et al., 2004).

\section{CONCEPTUAL DARE ARCHITECTURE DESCRIPTION}

Below we present an overview of the DARE concept, discuss the elements of the concept: planetary balloons, the Balloon Guidance System (BGS), and the deployable microprobes, and give examples of the applications at Venus, Titan and Jupiter.

\section{Concept Overview}

At the heart of the DARE concept are long-duration planetary balloons with trajectory control capabilities called DARE platforms. A conceptual drawing of the DARE platform is shown in Fig. 1. The figure shows a balloon with a gondola and a deployed Balloon Guidance System (BGS) Wing Assembly on a long tether below it. The drawing is for illustration purposes and is not to scale: the tether will be several $\mathrm{km}$ long and the BGS will be much smaller than the balloon. A larger view of the BGS is shown on Fig. 2a.

The BGS enables the DARE platform to be maneuvered relative to the prevailing atmospheric winds and permits targeted observations according to the mission objectives. The BGS requires very little power (about $1 \mathrm{~W}$ on average) to operate and can be made very light. It is positioned below the gondola on a long (several $\mathrm{km}$ ) tether. Details about the BGS can be found in the "Balloon Guidance System (BGS)" section of the paper. The DARE platform employs a superpressure balloon on Venus and Titan, and a Solar Infrared Montgolfier balloon on Jupiter (see section "Planetary Balloons"), remains aloft for about 100 days and is able to visit different regions of a planet. Several DARE platforms can form a constellation and perform simultaneous observations over a planet. The gondola on the DARE platform will carry several small, lightweight, deployable microprobes that can be released over target sites to perform in situ analysis of the atmosphere or surface (see section "Precision Deployable Microprobes"). The gondola also houses scientific instruments, computers, the BGS deployment system (for example, a lightweight winch), solar panels, batteries and antennas for communication with deployed microprobes and with the relay orbiter. Solar panels of reasonable sizes will not be able to provide enough power for DARE platforms at Jupiter and Titan. There, the DARE platforms will rely on Radioisotope Thermoelectric Generators (RTGs) to power and to heat the instrumentation on board. The role of the orbiter in this concept is primarily to relay the data gathered by the DARE platform and deployed microprobes back to Earth. Using an orbital relay means that the communications systems of the DARE platforms and deployable microprobes can be made relatively small, reducing the size of the balloons, the power requirements and the masses of the platforms and the microprobes. Several mission configurations can be envisioned, 1) the DARE platform being deployed at the planet that already has an orbiter from a different mission; 2) the orbiter and the DARE platform carried by the same spacecraft as two separate but synergistic missions; and 3) the DARE mission flight system carrying its own dedicated small communication orbiter.

Preliminary numerical analysis indicates that a relatively small $\left(1 \mathrm{~m}^{2}\right)$ BGS wing is capable of moving a balloon platform with the velocity of the order of $1 \mathrm{~m} / \mathrm{s}$ in the direction perpendicular to the direction of the winds at the balloon altitude (crosswind) at Venus and Titan. A larger BGS wing $\left(10 \mathrm{~m}^{2}\right)$ will be required at Jupiter to provide the same level of crosswind control velocity. The small but constantly applied offset can result in quite significant changes in a balloon trajectory. The trajectory change will happen slowly, but swift control actions will not be necessary. Preliminary analysis of the balloon guidance capabilities of the DARE platforms and example trajectories can be found in the "Applications and Trajectories Simulations at Venus, Titan and Jupiter" section of the paper. 
The DARE platforms can provide spatial coverage comparable to that of satellites, but they additionally provide capabilities for in situ atmospheric and surface analysis with deployable microprobes and high-resolution surface imaging. Floating much closer to the surface of the planet than an orbiter they can provide a wealth of new and unique observations. In the past the inability to control the balloons' paths has limited the interest in their usefulness. Without flight path guidance, a planetary balloon will quickly be carried away from a particular study region by the atmospheric winds and will never return to the same location. The BGS of the DARE platforms will permit targeting of preselected sites and revisiting them several times. The DARE BGS can vastly expand the capabilities for planetary exploration by providing the means to control paths of balloons in the planetary atmospheres. Examples of the DARE architectures deployment options and applications at Venus, Titan and Jupiter are given in the "Applications and Trajectories Simulations at Venus, Titan and Jupiter" section of the paper.

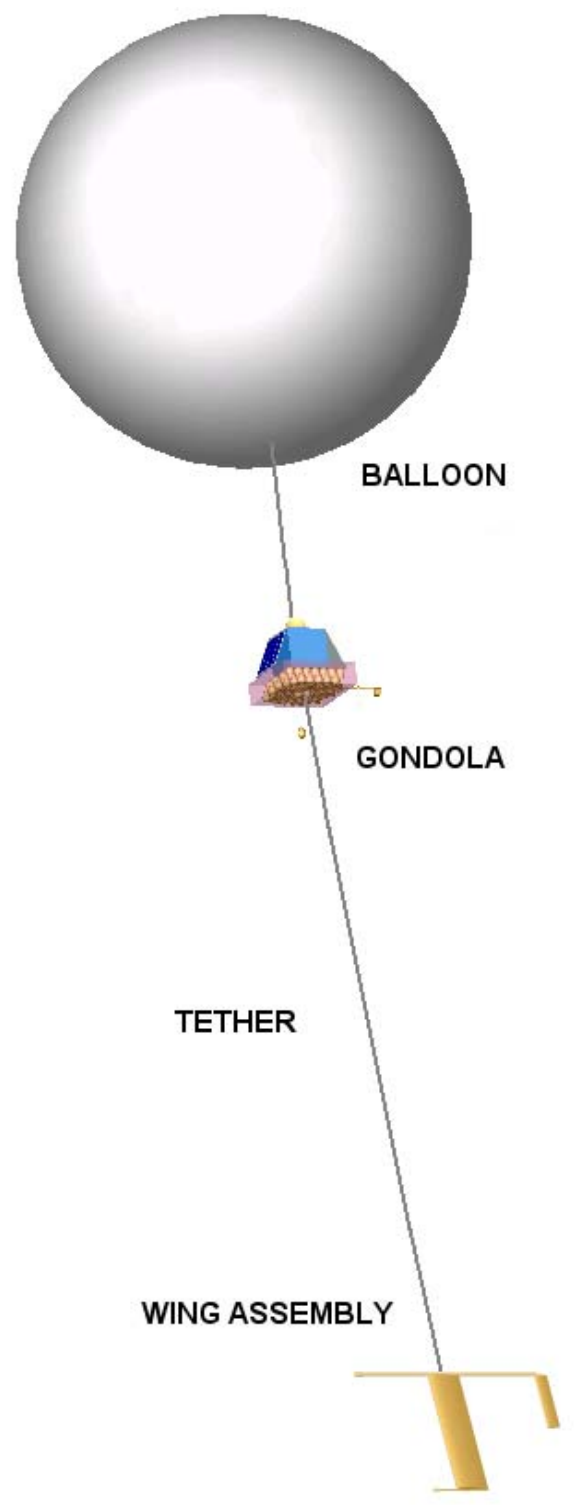

FIGURE 1. Conceptual View of the DARE Platform.

\section{Planetary Balloons}

Concepts for planetary balloons have been developed previously. We base our preliminary analysis of the DARE architecture on existing planetary balloon concepts. The balloon sizes were increased using a simple sizing model to 
account for the added mass of the BGS. In this preliminary analysis the masses of the deployable microprobes were included only in the Venus DARE analysis. Below we review the planetary balloon concepts that served as reference designs in assessing the BGS capabilities.

A Venus superpressure balloon concept was developed at NASA JPL in 1997 under the Discovery Program (JPL, 1997). The mission concept envisioned three helium superpressure balloons delivered by a single spacecraft. The balloons would be deployed at $54 \mathrm{~km}$ altitude at three different latitudes for a 30 to 100-day mission. Each balloon carried a $34 \mathrm{~kg}$ gondola and 4 dropsondes weighing $2.5 \mathrm{~kg}$ each. The concept envisioned direct communication with Earth via X-band antenna.

An analysis of a superpressure balloon performance at Titan has been performed by Science Applications International Corporation, (SAIC, 1983). In this analysis the floating altitude of the balloon versus mass of buoyant station and options for science payload were studied. The total system mass for a $100 \mathrm{~kg}$ payload was estimated at about $200 \mathrm{~kg}$ for floating altitudes from 0 to $50 \mathrm{~km}$, increasing to $400 \mathrm{~kg}$ for a floating altitude of $100 \mathrm{~km}$.

Solar Infrared Montgolfier Aerobots (SIRMAs) were proposed for studies of Jupiter and the Outer Planets (Jones and Heun, 1997). SIRMAs are "hot air" balloons that use planetary infrared heating during the night and solar heating during the day to remain afloat. SIRMA at Jupiter would float at about 0.1 bar during the day and descend to about 0.2 bar at night. A total floating mass of $112 \mathrm{~kg}$ would be required to suspend a $10-\mathrm{kg}$ payload. The total mass of the balloon assembly in this case would be an order of magnitude lighter than that of the comparable light-gas (pure hydrogen) balloon system at Jupiter. At Saturn a similar SIRMA system would have a mass of about $220 \mathrm{~kg}$, although this weight could be reduced in half if the SIRMA is flown during summer at a Saturn pole. There is not enough solar energy for a Montgolfier balloon at Uranus and Neptune. However, the upper methane-free stratospheric atmospheres of these planets have a significantly lower molecular weight than the atmosphere below the upper methane clouds. This unique feature makes it possible for relatively small balloons $(15 \mathrm{~kg})$ to fill with upper atmospheric gas and then float 50-kg payloads in the troposphere (Jones, 2001). In this study we only considered a Jupiter SIRMA.

\section{Balloon Guidance System (BGS)}

The BGS of the DARE platforms consists of a wing hanging on its side below the balloon on a very long (several $\mathrm{km}$ ) tether. Due to the difference in winds between the altitudes of the balloon and the wing, the wing experiences relative winds that create a sideways lifting force that can be used to pull the balloon across the winds. Because the density of the atmosphere is higher at the wing altitude, the wing can be much smaller than the balloon. Fig. 2 shows the first generation StratoSail ${ }^{\circledR}$ BGS and the advanced BGS currently being developed by Global Aerospace Corporation, respectively.

The advanced system increases the amount of control force (compared to the StratoSail ${ }^{\circledR}$ BGS) that can be exerted on a balloon in situations when the lift force is larger than the wing weight.

One of the advantages of the proposed BGS is that it does not require power for propulsion. A small amount of power ( $\sim 1 \mathrm{~W}$ on the average) is needed only for communications and to adjust the control surfaces of the guidance system once a day, or even less frequently, depending on the path control objectives. This power can be provided by a small solar panel attached to the surface of the BGS at Venus or wind turbines attached to the tether at Titan and Jupiter. Alternative approaches to balloon path guidance employing, for example, an engine driven propeller would require significantly higher levels of continuous power input $(\sim 1000 \mathrm{~W})$.

The BGS will be deployed from its stowage position on the gondola a short time after completion of the balloon inflation during atmospheric descent. The BGS will be folded and stowed below the gondola in the entry probe on the way to the planet. The deployment system may include a lightweight winch. The BGS will communicate with the control computer on the gondola via a radio link. The long tether can be also used to position lightweight pressure and temperature sensors along its length for atmospheric profiling, or magnetometers and gravimeters to study magnetic and gravity anomalies. 


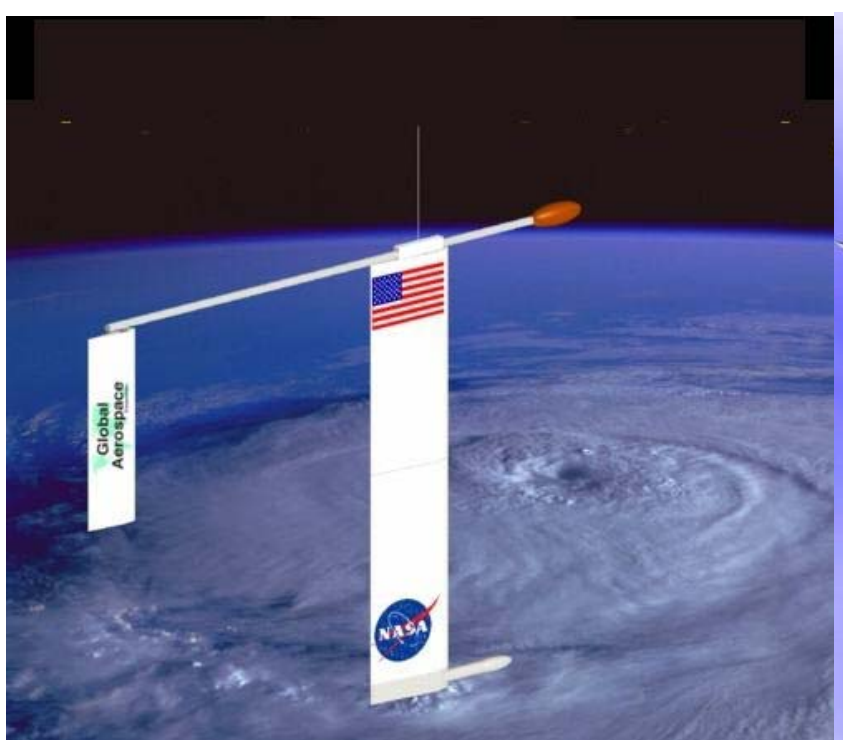

(a) StratoSail ${ }^{\circledR}$ BGS.

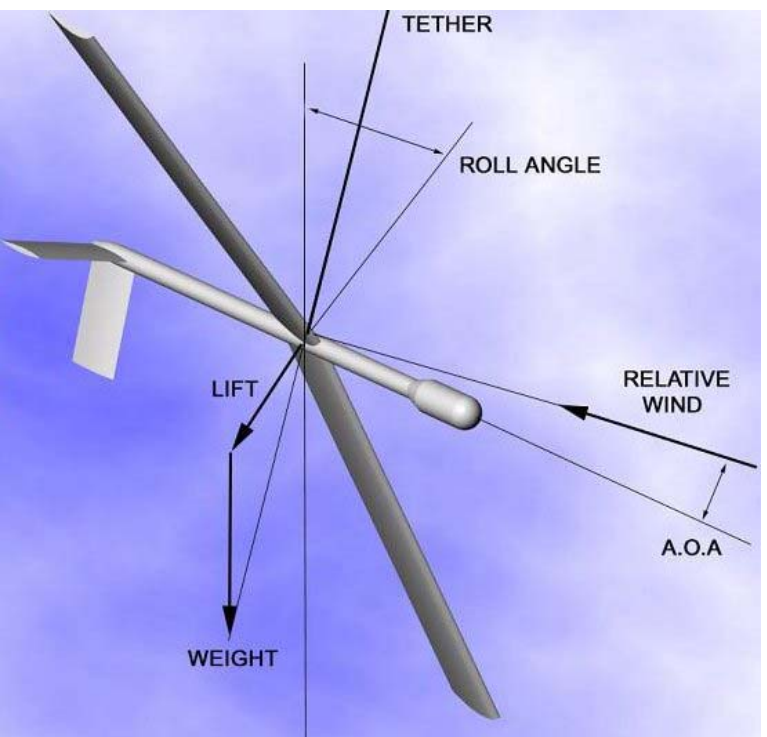

(b) Advanced BGS.

FIGURE 2. Balloon Guidance Systems (BGSs).

The examples of the StratoSail ${ }^{\circledR}$ BGS performance estimates for different planets and balloon reference designs are given in Table 1. The cross-track velocity is the balloon drift velocity due to the action of the BGS in the direction perpendicular to the wind direction at the balloon altitude. The numerical model of the BGS performance takes into account the change of the wing orientation in three dimensions due to the lift and drag forces, and the changes of the atmospheric density and the winds with altitude. These examples show that a relatively small BGS can provide about $1 \mathrm{~m} / \mathrm{s}$ of crosswind control velocity at Venus and Titan. The same level of control requires a much larger BGS at Jupiter. These seemingly small control velocities result in significant changes in balloon trajectories when applied continuously for many days. Results of trajectory simulations for different planets are presented in section "Applications and Trajectories Simulations at Venus, Titan and Jupiter" of the paper.

TABLE 1. Summary of the Balloon Guidance Capabilities for DARE Platforms at Venus, Titan and Jupiter.

\begin{tabular}{|c|c|c|c|}
\hline Parameter & Venus & Titan & Jupiter \\
\hline Balloon type & Superpressure & Superpressure & SIRMA \\
\hline Balloon float level & $54 \mathrm{~km}$ & $50-80 \mathrm{~km}$ & $0.1-0.2$ bar \\
\hline Balloon diameter & $7.6 \mathrm{~m}$ & $8.7-19.8 \mathrm{~m}$ & $72 \mathrm{~m}$ \\
\hline Wing area & $1 \mathrm{~m}^{2}$ & $1 \mathrm{~m}^{2}$ & $10 \mathrm{~m}^{2}$ \\
\hline Tether length & $10 \mathrm{~km}$ & $10 \mathrm{~km}$ & $10 \mathrm{~km}$ \\
\hline Wing mass* & $10 \mathrm{~kg}$ & $10 \mathrm{~kg}$ & $50 \mathrm{~kg}$ \\
\hline Tether mass* & $7.5 \mathrm{~kg}$ & $5 \mathrm{~kg}$ & $10 \mathrm{~kg}$ \\
\hline Total floating mass & $202 \mathrm{~kg} * *$ & $125-195 \mathrm{~kg}$ & $208 \mathrm{~kg}$ \\
\hline Wind shear & $1.6 \mathrm{~m} / \mathrm{s} / \mathrm{km}$ & $1 \mathrm{~m} / \mathrm{s} / \mathrm{km}$ & $1.5 \mathrm{~m} / \mathrm{s} / \mathrm{km}$ \\
\hline Cross-track velocity & $1.5 \mathrm{~m} / \mathrm{s}$ & $0.5-0.9 \mathrm{~m} / \mathrm{s}$ & $1.0 \mathrm{~m} / \mathrm{s}$ \\
\hline
\end{tabular}

* preliminary estimate based on Earth analog

** including $1001 \mathrm{~kg}$ dropsondes 


\section{Precision Deployable Microprobes}

A variety of deployable microprobe configurations are possible, able to transmit $\sim 1$ Mbits of data over an hour or so over several tens to one or two hundred $\mathrm{km}$, to the balloon, with masses of 0.1-1 kg. The scale involved suggests scope for imaginative thinking (Lorenz, 1998; Thakoor et al., 1999).

The delivery of microprobes from a balloon enables each microprobe to eliminate the usual entry protection and delivery systems (which can be a significant portion of the total entry mass), which opens up revolutionary possibilities for the configuration and size of the microprobes.

The smaller the size of the microprobe the more can be carried on a DARE mission. The small size necessitates a much higher level of integration. For example, rather than having a separate structure to which components are attached, the small size may mean the lifting surface (of a balloon envelope, or a wing in a flyer) acts as the structure. Similarly, while the small size may not permit traditional components and approaches, e.g. for tracking, there may be considerable economies of scale in improving analysis and calibration techniques - such as post facto temperature compensation for sensors by analysis and temperature history, rather than applying massive and powerhungry temperature controls to each sensor. An investigation of the frequency stability of radio links for miniaturized hardware, potentially with large temperature excursions as might be expected on a microprobe, would be an important part of assessing the scientific potential of microprobe Doppler tracking measurements. Ranging transponders on the microprobes, coupled with an interferometric antenna array on the balloon, may offer the best performance for realistic costs and robust implementation.

The small size (and cost) of each microprobe makes possible a wider involvement of educational institutions, since it becomes possible for each class or institution to build their own probe, either as an analogue, or even as one of the many microprobes to be released. This architecture therefore offers many educational, outreach and involvement opportunities.

\section{Applications and Trajectories Simulations at Venus, Titan and Jupiter}

Below we present preliminary analysis of the applications for the DARE architecture and simulations of the DARE platforms' trajectories at Venus, Titan and Jupiter. To simulate the trajectories we developed simplified models of the planetary atmospheres that prescribe zonal and meridional winds.

\section{Venus}

At Venus the DARE platform will float in the upper atmosphere (above $50 \mathrm{~km}$ ), above the Venus clouds. The platform will employ a superpressure balloon. Solar panels or wind turbines will be used to power the instruments and computers. The data from the microprobes and from the platform could be relayed directly to Earth by the DARE platform or to an orbiter. Examples of unique scientific observations that will become possible with the DARE architecture include:

- complete inventory of the atmospheric constituents with onboard mass spectrometers and UV and IR spectrometers;

- emplacement of a surface seismological and meteorological network (15 to 20 sites for meteorology, 8 or more sites for seismology) to the locations that span latitude, longitude, and elevation contrasts;

- targeted delivery of surface and subsurface probes that perform elemental, compositional, chemical and chronological studies at preselected sites;

- high-resolution imagery reconnaissance of potential spacecraft landing sites; an onboard high-resolution camera or a gliding probe provide images revealing 1 to $10 \mathrm{~cm}$ scale objects on the surface.

- monitoring global circulation with a constellation of several DARE platforms simultaneously; monitor winds and atmospheric parameters in different regions of the atmosphere (equatorial, midlatitude, polar). Another approach is to have a single platform visit different atmospheric regions. Single platform approach is suitable for Venus because a platform would be able to sample both day and night sides of the planet during one Venus day. 
The simplified model of the Venus atmospheric winds assumes zonal winds linearly increasing with altitude and meridional winds dominated by solar tides (Newman and Leovy, 1992).

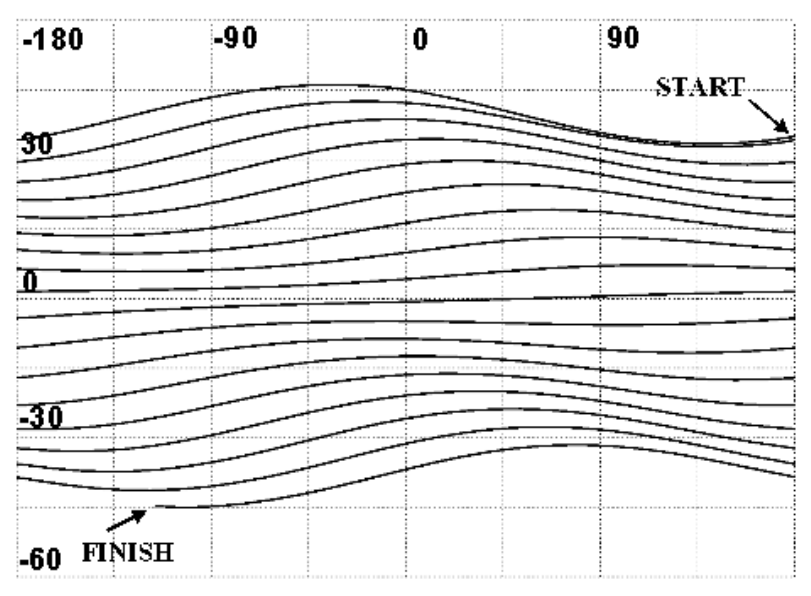

(a) Venus DARE Platform Trajectory at $54 \mathrm{~km}$.

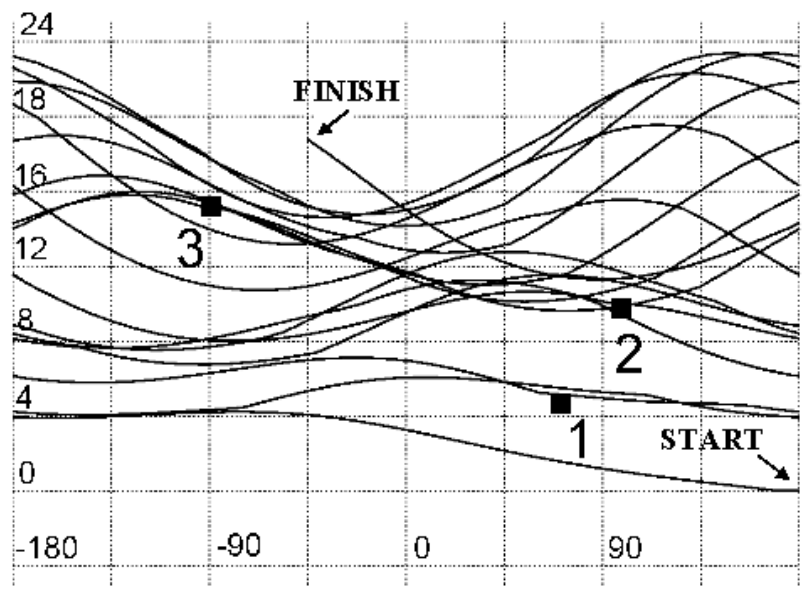

(b) Venus DARE Multiple Target Overflight.

FIGURE 3. Examples of the DARE Trajectories at Venus.

Fig. 3a shows the trajectory of a controlled balloon at Venus at $54 \mathrm{~km}$. The DARE platform configuration is as described in Table 1. Horizontal axis is longitude from $-180^{\circ}$ to $180^{\circ}$; vertical axis is latitude from $-60^{\circ} \mathrm{S}$ to $60^{\circ} \mathrm{N}$. The objective of the simulation is to analyze the coverage that can be achieved with the controlled balloon at Venus. The maximum control cross-track velocity is $1 \mathrm{~m} / \mathrm{s}$. The balloon starts at $180^{\circ} \mathrm{E}$ and $30^{\circ} \mathrm{N}$ and is directed south. During a 100-day simulation the balloon is able to visit latitudes between $45^{\circ} \mathrm{N}$ and $45^{\circ} \mathrm{S}$. The balloon completes almost 18 circumnavigations. The uncontrolled balloon would only be able to sweep a latitudinal corridor about $15^{\circ}$ wide during a 100-day mission.

Fig. 3b shows simulations of a target overflight. Horizontal axis is longitude from $-180^{\circ}$ to $180^{\circ}$; vertical axis is latitude from $-5^{\circ} \mathrm{S}$ to $25^{\circ} \mathrm{N}$. In this simulation the DARE platform is directed to fly over several surface targets. Three targets (indicated by 1, 2 and 3 on the figure) are "turned on" sequentially. Target 1 is "turned on" first; when the balloon flies over it target 1 is "turned off" and target 2 is "turned on", and so on. The latitudes of the target were chosen to span a range of latitudes. The longitudes were chosen randomly. The DARE platform can be directed to fly very close to a target. In this simulation the balloon is directed to fly within $25 \mathrm{~km}$ of the targets. The balloon is started at the equator at $180^{\circ} \mathrm{E}$ and is able to fly over 3 targets during the 100-day simulation. It takes 3,4 and 8 circumnavigations to fly over targets 1, 2 and 3, respectively. It is easier to perform targeting closer to the equator (target 1), where meridional winds are weak and comparable to the cross-track velocity provided by the BGS (1 $\mathrm{m} / \mathrm{s}$ ). In the midlatitudes (target 3 ) the meridional velocity is the largest and thus effective platform control is the weakest. Another simulation (not shown) indicates that four point targets in midlatitudes can be overflown within $150 \mathrm{~km}$ (horizontal distance) during a 100-day simulation.

Titan

After the reconnaissance flyby of Titan by the Cassini spacecraft and the deployment of the Huygens probe, the next logical exploration step would be the global mapping of Titan. As with the exploration of the planets, where telescopic observations and reconnaissance flybys (Mariners, Pioneers, Voyagers) were followed by global exploration orbiters (Magellan at Venus, and Mariner 9, Vikings, Mars Global Surveyor, Odyssey at Mars) and then by focused exploration (Viking Landers, Pathfinder, Mars Exploration Rover Mission at Mars), the next mission to Titan should provide a global view of the planet from an orbiter. However, the thick organic haze covering Titan will block a wide range of the useful spectrum for the instruments deployed on space orbiters. The DARE architecture with its platforms deployed below the haze is best suited for such an atmospheric "orbital" mission. 
Altitudes above $50 \mathrm{~km}$ and below $80 \mathrm{~km}$ seem to be best suited for the DARE platform operations at Titan. This is the lower stratosphere of Titan, situated beneath most of the organic haze. The region is characterized by strong zonal winds, in excess of $50 \mathrm{~m} / \mathrm{s}$ (Flasar et al., 1997), so circumnavigation takes about 3.5 days at the equator, less at higher latitudes. The DARE platform will probably employ RTGs or wind turbines to power and heat the instruments and computers. The architecture will require an orbiter to relay the data to Earth. Unique observations at Titan include:

- mapping, weather monitoring and change detection with wide-area down-looking imager; search for methane lakes and seas;

- targeted deployment of biochemical analyzers, rovers and floaters;

- measurement of the winds as a function of altitude, location via microprobe deployment, autonomous navigation and/or tracking from ground or orbiter;

- measurement of gas abundances as a function of altitude, location;

- studies of the condensation processes in the atmosphere;

- surface studies with microprobes, including surface chemistry analyzers and sample acquisition systems, radiation sensors to carbon-date surface deposits, seismometers.

The simplified model of the Titan atmospheric winds assumes zonal winds from Flasar et al. (1997) with the meridional winds from Tokano and Neubauer (2002). The meridional winds are very weak - about $2 \mathrm{~m} / \mathrm{s}$ at these altitudes so that a free-floating balloon would stay in a very narrow latitudinal corridor during its flight. On the contrary, the controlled balloon is able to achieve almost global coverage during a 100-day mission. Fig. 4 shows the trajectory of the DARE platform at Titan at $60 \mathrm{~km}$ altitude. The maximum BGS control velocity in this simulation is $0.9 \mathrm{~m} / \mathrm{s}$ (platform configuration is as described in Table 1 ).

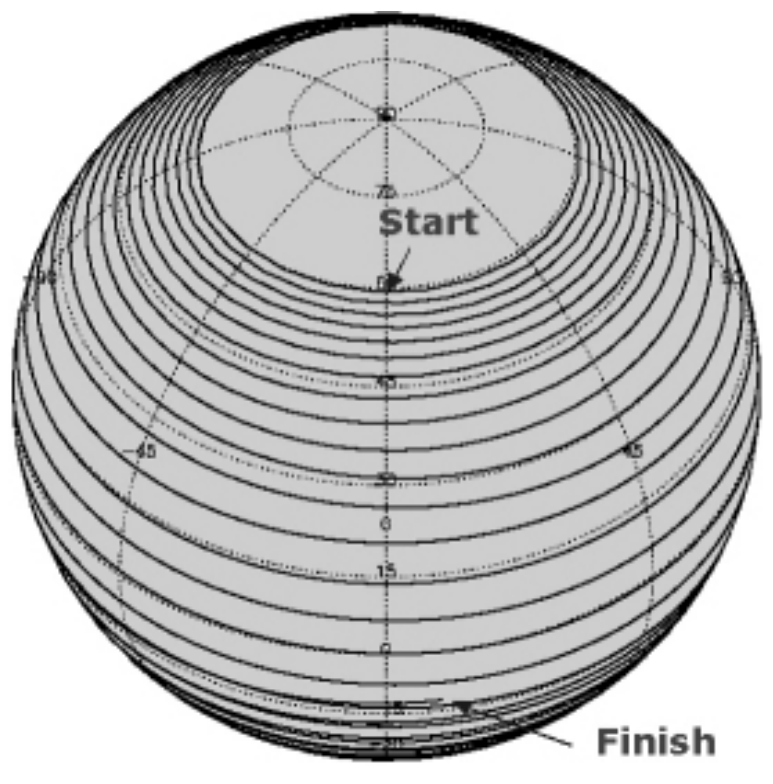

FIGURE 4. Titan DARE Trajectory at $60 \mathrm{~km}$.

The balloon is started at $180^{\circ} \mathrm{E}$ and $60^{\circ} \mathrm{N}$. The balloon is directed to "sweep" the corridor between $60^{\circ} \mathrm{N}$ and $60^{\circ} \mathrm{S}$. After touching $60^{\circ} \mathrm{N}$ it is directed south. After reaching $60^{\circ} \mathrm{S}$ it is directed north again. By the end of the 100-day mission the balloon is able to reach the $60^{\circ} \mathrm{S}$ and to return to about $10^{\circ} \mathrm{S}$. This analysis shows that balloon control with the proposed BGS is sufficient to observe most of the planet over a 100-day mission and greatly expands the capabilities of a single balloon. 


\section{Jupiter}

At Jupiter, the DARE platform would employ a SIRMA balloon and float at the pressure level of 0.1-0.2 bar (above the methane cloud level). The DARE platform will probably employ RTGs or wind turbines to power and heat the instruments and computers. Unique observations at Jupiter include:

- deployment of probes to study atmospheric circulation and composition above and below cloud decks, inside the zones and bands of zonal winds;

- targeted observations of localized regions of interest, such as "hot spots" (like the one encountered by the Galileo probe) and large vortices, like the Great Red Spot (GRS).

The simple model of the Jupiter winds is based on the zonal winds profile (Limaye, 1986) with the superimposed synthetic wind field of the Great Red Spot (GRS) (Dowling and Ingersoll, 1988). The winds decay with altitude above the cloud level with the decay scale height equal to about $60 \mathrm{~km}$.

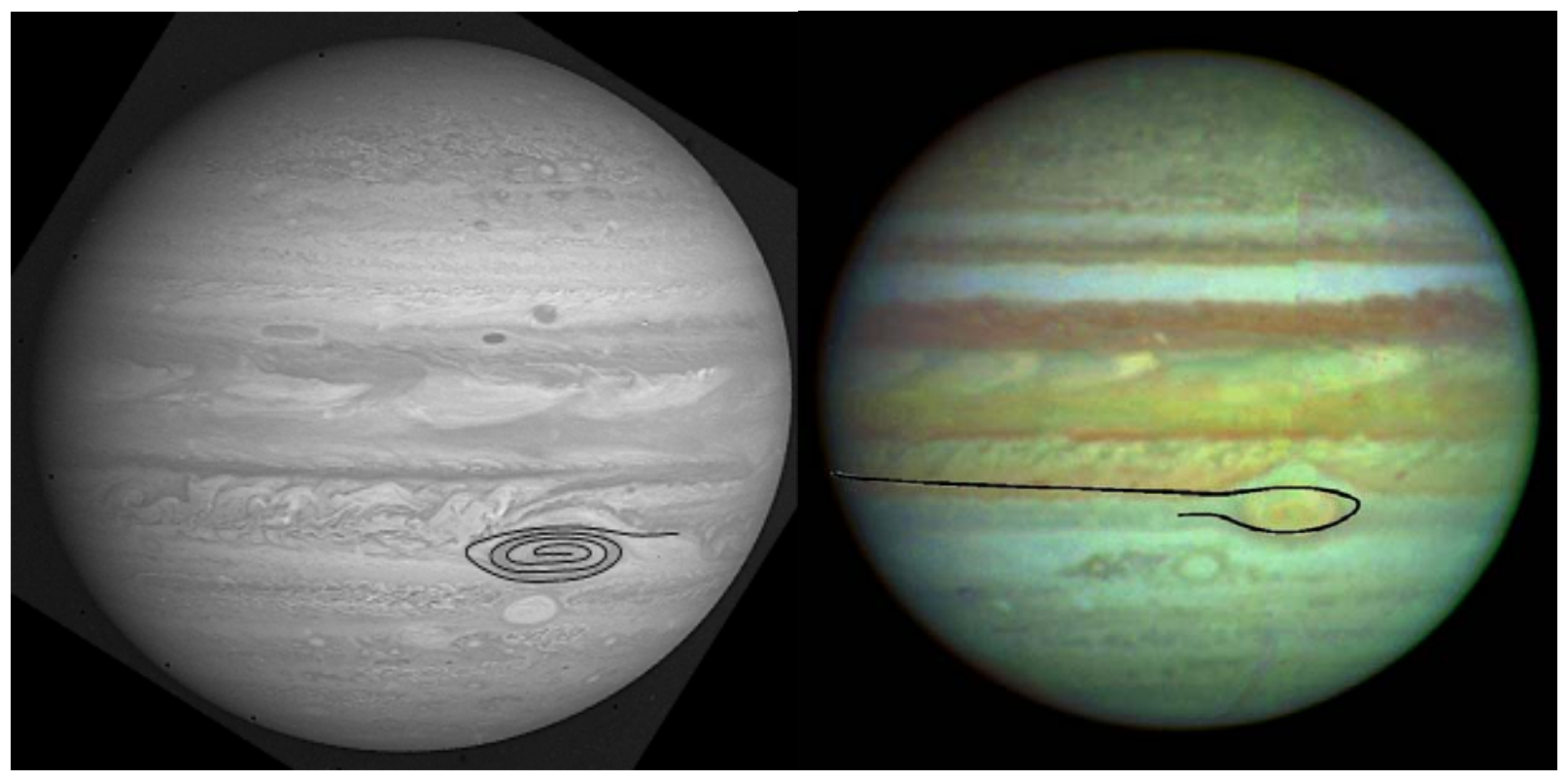

(a) DARE Platform Spiraling into the GRS.

(b) DARE Platform Crossing a Belt and a Zone.

FIGURE 5. Examples of the DARE Trajectories at Jupiter.

The goal of the simulation is to see how much of the GRS can be sampled by a DARE platform during a 100-day mission. Fig. 5a shows the trajectory of the DARE platform spiraling into the GRS plotted over an image of Jupiter. The maximum BGS control velocity is $0.9 \mathrm{~m} / \mathrm{s}$. The BGS pushes the DARE platform toward the center of the GRS. The balloon is able to reach the center of the GRS after about 4 revolutions around the GRS. The DARE platform deploys probes in the process, thus sampling different regions of the GRS. In another example illustrating the advantages of balloon guidance capabilities of the DARE platforms shown in Fig. 5b, the goal of the simulation is to observe large parts of the neighboring belt and zone. The DARE platform trajectory is indicated by a heavy black curve plotted over an image of Jupiter. In this simulation the BGS guides the DARE platform towards the GRS in an attempt to use strong winds inside the GRS to significantly change the balloon trajectory. The meridional winds inside the GRS are much stronger $(100 \mathrm{~m} / \mathrm{s})$ than the velocity provided by the BGS, thus, if the balloon can enter the northward or southward branch of the GRS and quickly exit it, the traveled latitudinal distance can be much greater than without the GRS assistance. The DARE platform in Fig. 5b is started to the west from the GRS and is pushed northward by the BGS. The atmospheric winds carry the balloon eastward, then around the GRS and then westward. The total traveled latitudinal distance is about $8^{\circ}$. The DARE balloon is able to travel from about the middle of the zone to the middle of the belt, thus accomplishing the simulation objective. It is possible to cross from the belt into a zone (or visa versa) using the BGS without the assistance of the GRS, but the traveled latitudinal distance would be 
smaller (by about 25\%). The latitudinal distance traveled with the assistance of the GRS would be greater with a more efficient BGS.

\section{CONCLUSIONS}

A concept for a new architecture for planetary exploration is proposed. The key elements of the architecture are: long-duration-flight autonomous balloons, balloon trajectory control, lightweight power generation and storage, and multiple deployable microprobes for atmosphere and surface exploration. A relatively small, light balloon trajectory control device would enable repositioning of the platform on a global scale for in situ analysis and targeted deployment of atmospheric and surface probes. Deployment of microprobes from balloons eliminates atmospheric entry and deceleration hardware thus reducing probes mass and permitting more science payloads or more probes. Miniaturization of probes offers innovative approaches to in situ and remote observations. The DARE architecture will enable low-cost, low-energy, long-term global exploration of the atmosphere and surface of Venus, Titan, Mars and Jupiter. Additional information can be found at http://www.gaerospace.com/projects/DARE/DARE.html.

\section{ACKNOWLEDGMENTS}

This work was supported by the NASA Institute for Advanced Concepts (NIAC) under USRA Contract no. 07600099.

\section{REFERENCES}

Bachelder, A. et al., "Venus Geoscience Aerobot Study," AIAA paper 99-3856, 1999.

Cutts, J.A. et al., "Venus Aerobot Multisonde Mission," AIAA Balloon Tech. Conference, 1-10, 1999.

Dowling, T. E. and Ingersoll, A. P., "Potential vorticity and thickness variations in the flow around Jupiter Great Red Spot and White Oval BC," J. of Atmospheric Science, 45(8), 1380-1396 (1988).

Flasar, F. M., Allison, M. D. and Lunine, J. I., "Titan zonal wind model," in Huygens: Science, Payload and Mission, edited by A. Wilson, ESA SP-1177, 1997, pp. 297-298.

Greeley, R. et al., "The Mars Aerial Platform Mission Concept,” AIAA paper 96-0335, 1996.

Jones, J. A. and Heun, M. K., “Montgolfier Balloon Aerobots for Planetary Atmospheres,” AIAA, Paper 97-1445, 1997.

Jones, J. A. and Wu, J., "Solar Montgolfier Balloons for Mars," AIAA paper 99-3852, 1999.

Jones, J. A., "Inflatable Vehicles for In-Situ Exploration of Titan, Triton, Uranus, Neptune," Forum on Innovative Approaches to Outer Planetary Exploration, Lunar and Planetary Institute, Houston, Texas, February 21-22, 2001.

JPL, "Venus balloon Discovery study," informal JPL briefing, September 23, 1997.

Limaye S. S., "Jupiter: New estimates of the mean zonal flow at the cloud level," Icarus, 65 (1986).

Lorenz, R. D., "Design Considerations for Venus Microprobes," J. of Spacecraft and Rockets, 35(2), $228-230$ (1998).

Newman, M., and Leovy, C., "Maintenance of strong rotational winds in Venus' middle atmosphere by thermal tides," Science, 257, 647-650 (1992).

Nock, K.T. et al., "Overview of a Mars 2001 Aerobot/Balloon System," 12th AIAA Lighter-Than-Air Technology Conference, San Francisco, 1997.

Pankine A. et al., "Mars Exploration with Directed Aerial Robot Explorers," in these Proceedings of Space Technology and Application International Forum (STAIF-2004), edited by M. S. El-Genk, American institute of Physics, Melville, NY, 2004.

Sagdeev, R. Z. et al., "Overview of the VEGA Venus balloon in situ meteorological measurements," Science, 231, 1411-1422 (1986).

SAIC, "Titan exploration with advanced systems, a study of future mission concepts", NASA CR-173499, 1983.

Tarrieu, C., "Status of the Mars 96 Aerostat Development," 44th Congress of the International Astronautical Federation, paper IAF-93-Q.3.399, 1993.

Tokano, T. and Neubauer, F., "Tidal winds on Titan caused by Saturn," Icarus, 158, 499-515 (2002).

Thakoor, S. et al., "Cooperative mission concepts using biomorphic explorers," in Lunar and Planetary Science XXX, paper 2029, 1999

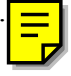

